PROCEEDINGS OF THE

AMERICAN MATHEMATICAL SOCIETY

Volume 136, Number 6, June 2008, Pages 2261-2270

S 0002-9939(08)09284-8

Article electronically published on January 28, 2008

\title{
A GENERATING FUNCTION FOR BLATTNER'S FORMULA
}

\author{
JEB F. WILLENBRING AND GREGG J. ZUCKERMAN
}

(Communicated by Gail R. Letzter)

\begin{abstract}
Let $G$ be a connected, semisimple Lie group with finite center and let $K$ be a maximal compact subgroup. We investigate a method to compute multiplicities of $K$-types in the discrete series using a rational expression for a generating function obtained from Blattner's formula. This expression involves a product with a character of an irreducible finite-dimensional representation of $K$ and is valid for any discrete series system. Other results include a new proof of a symmetry of Blattner's formula, and a positivity result for certain low rank examples. We consider in detail the situation for $G$ of type split $\mathrm{G}_{2}$. The motivation for this work came from an attempt to understand pictures coming from Blattner's formula, some of which we include in the paper.
\end{abstract}

\section{INTRODUCTION}

In [7, a proof of a formula for the restriction of a discrete series representation (see [6]) of a connected, linear, semisimple Lie group to a maximal compact subgroup is given. This formula was first conjectured by Blattner. We recall the formula and its context briefly, from the point of view of root system combinatorics.

Throughout the paper, $\mathfrak{g}$ denotes a semisimple Lie algebra over $\mathbb{C}$ with a fixed Cartan subalgebra $\mathfrak{h}$. Let $\Phi:=\Phi(\mathfrak{g}, \mathfrak{h})$ denote the corresponding root system with Weyl group $W_{\mathfrak{g}}$. Choose a set, $\Phi^{+}$, of positive roots and let $\Pi:=\left\{\alpha_{1}, \ldots, \alpha_{r}\right\} \subseteq \Phi$ be the simple roots. Let $\Phi^{-}=-\Phi^{+}$.

We assume that there exists a function $\theta: \Phi \rightarrow \mathbb{Z}_{2}$ such that if $\gamma_{1}, \gamma_{2} \in \Phi$ and $\gamma_{1}+\gamma_{2} \in \Phi$, then $\theta\left(\gamma_{1}+\gamma_{2}\right)=\theta\left(\gamma_{1}\right)+\theta\left(\gamma_{2}\right)$. This map provides a $\mathbb{Z}_{2}$-gradation on $\Phi$. We set:

$$
\begin{aligned}
& \Phi_{c}:=\{\gamma \in \Phi \mid \theta(\gamma)=0\} \\
& \Phi_{n c}:=\{\gamma \in \Phi \mid \theta(\gamma)=1\}
\end{aligned}
$$

Given $\alpha \in \Phi$, set $\mathfrak{g}_{\alpha}=\{X \in \mathfrak{g} \mid[H, X]=\alpha(H) X \forall H \in \mathfrak{h}\}$. Let $\mathfrak{k}:=\mathfrak{h} \oplus \sum_{\alpha \in \Phi_{c}} \mathfrak{g}_{\alpha}$ and $\mathfrak{p}:=\sum_{\alpha \in \Phi_{n c}} \mathfrak{g}_{\alpha}$. Then, $\mathfrak{k}$ will be a reductive symmetric subalgebra of $\mathfrak{g}$ with $\mathfrak{g}=\mathfrak{k} \oplus \mathfrak{p}$ the corresponding Cartan decomposition of $\mathfrak{g}$. As defined, $\mathfrak{h}$ is a Cartan subalgebra for $\mathfrak{k}$, so rank $\mathfrak{k}=$ rank $\mathfrak{g}$. Each equal rank symmetric pair corresponds to at least one $\mathbb{Z}_{2}$-gradation in this manner, and conversely.

We shall refer to the elements of $\Phi_{c}\left(\right.$ resp. $\left.\Phi_{n c}\right)$ as compact (resp. noncompact). The compact roots are a subroot system of $\Phi$. Let $\Phi_{c}^{+}:=\Phi^{+} \cap \Phi_{c}, \Phi_{n c}^{+}:=\Phi^{+} \cap \Phi_{n c}$,

Received by the editors April 26, 2007.

2000 Mathematics Subject Classification. Primary 22E46; Secondary 17B10.

Key words and phrases. Blattner's formula, coherent continuation, discrete series.

The first author was supported in part by NSA Grant \# H98230-05-1-0078.

(C)2008 American Mathematical Society 
$\Pi_{c}:=\Pi \cap \Phi_{c}$, and $\Pi_{n c}:=\Pi \cap \Phi_{n c}$. Set $\rho_{\mathfrak{g}}:=\rho_{c}+\rho_{n c}$ where $\rho_{c}:=\frac{1}{2} \sum_{\alpha \in \Phi_{c}^{+}} \alpha$ and $\rho_{n c}=\frac{1}{2} \sum_{\alpha \in \Phi_{n c}^{+}} \alpha$. If there is no subscript, we mean $\rho=\rho_{c}$.

We remark that the $\mathbb{Z}_{2}$-gradation $\theta$ is determined by its restriction to $\Pi$. Furthermore, to any set partition $\Pi=\Pi_{1} \biguplus \Pi_{2}$ there exists a unique $\mathbb{Z}_{2}$-gradation on $\Phi$ such that $\Pi_{c}=\Pi_{1}$ and $\Pi_{n c}=\Pi_{2}$.

We denote the Killing form on $\mathfrak{g}$ by $($,$) , which restricts to a nondegenerate$ form on $\mathfrak{h}$. Using this form we may define $\iota: \mathfrak{h} \rightarrow \mathfrak{h}^{*}$ by $\iota(X)(-)=(X,-)$ $(X \in \mathfrak{h})$, which allows us to identify $\mathfrak{h}$ with $\mathfrak{h}^{*}$. Under this identification, we have $\iota\left(H_{\alpha}\right)=\frac{2 \alpha}{(\alpha, \alpha)}=: \alpha^{\vee}$, where $H_{\alpha} \in \mathfrak{h}$ is the simple coroot corresponding to $\alpha \in \Pi$.

For each $\alpha \in \Phi$, set $s_{\alpha}(\xi)=\xi-\left(\xi, \alpha^{\vee}\right) \alpha\left(\right.$ for $\left.\xi \in \mathfrak{h}^{*}\right)$ to be the reflection through the hyperplane defined by $\alpha^{\vee}$. For $\alpha_{i} \in \Pi$, let $s_{i}:=s_{\alpha_{i}}$ be the simple reflection defined by $\alpha_{i}$. Define $\Pi_{\mathfrak{k}}$ to be the set of simple roots in $\Phi_{c}^{+}$and let $W_{\mathfrak{k}}$ denote the Weyl group generated by the reflections defined by $\Pi_{\mathfrak{k}}$. Let $W_{c}=\left\langle s_{\alpha} \mid \alpha \in \Pi_{c}\right\rangle$ be the parabolic subgroup of $W_{\mathfrak{g}}$ defined by the compact simple $\mathfrak{g}$-roots. Note that $W_{c} \subseteq W_{\mathfrak{k}}$, but we do not have equality in general. For $w \in W_{\mathfrak{k}}$, set $\ell(w):=$ $\left|w\left(\Phi_{c}^{+}\right) \cap \Phi_{c}^{-}\right|$. Note that there is also a length function on $W_{\mathfrak{g}}$ (denoted by $\ell_{\mathfrak{g}}$ ) but $\ell$ refers to $W_{\mathfrak{k}}$.

A weight $\xi \in \mathfrak{h}^{*}$ is said to be $\mathfrak{k}$-dominant (resp. $\mathfrak{g}$-dominant) if $(\xi, \alpha) \geq 0$ for all $\alpha \in \Pi_{\mathfrak{k}}(\operatorname{resp} \alpha \in \Pi)$. A weight $\xi \in \mathfrak{h}^{*}$ is $\mathfrak{g}$-regular (resp. $\mathfrak{k}$-regular) if $(\xi, \alpha) \neq 0$ for all $\alpha \in \Phi$ (resp. $\alpha \in \Phi_{c}$ ). The integral weight lattice for $\mathfrak{g}$ is denoted by the set $P(\mathfrak{g})=\left\{\xi \in \mathfrak{h}^{*} \mid\left(\xi, \alpha^{\vee}\right) \in \mathbb{Z}\right.$ for all $\left.\alpha \in \Pi_{\mathfrak{g}}\right\}$. Similarly we let $P(\mathfrak{k})$ denote the abelian group of integral weights for $\mathfrak{k}$ corresponding to $\Pi_{\mathfrak{k}}$. Let the set of $\mathfrak{k}-$ and $\mathfrak{g}$-dominant integral weights be denoted by $P_{+}(\mathfrak{k})$ and $P_{+}(\mathfrak{g})$ respectively. To each element $\delta \in P_{+}(\mathfrak{k})$ (resp. $P_{+}(\mathfrak{g})$ ), let $L_{\mathfrak{k}}(\delta)$ (resp. $\left.L_{\mathfrak{g}}(\delta)\right)$ denote the finitedimensional representation of $\mathfrak{k}$ (resp. $\mathfrak{g}$ ) with highest weight $\delta$.

Next, let $Q: P(\mathfrak{k}) \rightarrow \mathbb{N}$ denote the $\Phi_{n c}^{+}$-partition function. That is, if $\xi \in \mathfrak{h}^{*}$, then $Q(\xi)$ is the number of ways of writing $\xi$ as a sum of noncompact positive roots. Put another way, $Q$ defines the coefficients of the product:

$$
\sum_{\xi \in \mathfrak{h}^{*}} Q(\xi) e^{\xi}=\prod_{\gamma \in \Phi_{n c}^{+}}\left(1-e^{\gamma}\right)^{-1}
$$

Finally, we define the Blattner formula. For $\delta, \mu \in P(\mathfrak{k})$,

$$
B(\delta, \mu):=\sum_{w \in W_{\mathbf{k}}}(-1)^{\ell(w)} Q(w(\delta+\rho)-\rho-\mu) .
$$

It is convenient to introduce the notation $w \cdot \xi=w(\xi+\rho)-\rho$ for $w \in W_{\mathfrak{k}}$ and $\xi \in \mathfrak{h}^{*}$. It is easy to see that $B(v . \delta, \mu)=(-1)^{\ell(v)} B(\delta, \mu)$. In light of this fact, we assume that $\delta \in P_{+}(\mathfrak{k})$.

Historically, Blattner's formula arises out of the study of the discrete series and its generalizations (see [1,3, 5, 7, 10, 13]).

Theorem 1.1 (see [7). Assume $\lambda=\lambda(\mu):=\mu-\rho_{n c}+\rho_{c}$ is $\mathfrak{g}$-dominant and $\mathfrak{g}$ regular. Then, $B(\delta, \mu)$ is the multiplicity of the finite-dimensional $\mathfrak{k}$-representation, $L_{\mathfrak{k}}(\delta)$, in the discrete series representation of $G$ with Harish-Chandra parameter $\lambda$.

In this paper, we do not impose the $\mathfrak{g}$-dominant regular condition on $\lambda(\mu)$. This is natural from the point of view of representation theory as it is related to the coherent continuation of the discrete series (see [10], [1] and [14]). 
From our point of view, the goal is to understand the Blattner formula in as combinatorial a fashion as possible. Thus it is convenient to introduce the following generating function:

Definition. For $\delta \in P_{+}(\mathfrak{k})$ we define the formal series:

$$
\mathbf{b}(\delta):=\sum_{\mu \in \mathfrak{h}^{*}} B(\delta, \mu) e^{\mu} .
$$

The main result of this paper is Proposition 2.1 of Section 2, which states: For $\delta \in P_{+}(\mathfrak{k})$,

$$
\mathbf{b}(\delta)=\operatorname{ch} L_{\mathfrak{k}}(\delta) \frac{\prod_{\gamma \in \Phi_{c}^{+}}\left(1-e^{-\gamma}\right)}{\prod_{\gamma \in \Phi_{n c}^{+}}\left(1-e^{-\gamma}\right)},
$$

where $\operatorname{ch} L_{\mathfrak{k}}(\delta)$ denotes the character of $L_{\mathfrak{k}}(\delta)$.

Of particular interest are the cases where $\Pi_{c} \neq \emptyset$, which we address in Section 3. From the point of view of representation theory these include, for example, the holomorphic and Borel-de Siebenthal discrete series (see [7]). More recently, the latter has been addressed in 5 .

The Blattner formula for the case of $\Pi_{c}=\emptyset$ is often particularly difficult to compute explicitly when compared to, say, the cases corresponding to holomorphic discrete series. The $\Pi_{c}=\emptyset$ case corresponds to the generic discrete series of the corresponding real semisimple Lie group. In Section 4 we explore this situation in some detail for the Lie algebra $\mathrm{G}_{2}$.

Finally, in light of Theorem 1.1 one may observe that if $\delta \in P_{+}(\mathfrak{k})$ and $\lambda(\mu)$ is $\mathfrak{g}$-dominant regular, then $B(\delta, \mu) \geq 0$. Our goal is to investigate the positivity of Blattner's formula using combinatorial methods. Of particular interest is the positivity when we relax the $\mathfrak{g}$-dominance condition on $\lambda(\mu)$. Some results in this direction are suggested by the recent work of Penkov and the second author (see [9]).

In Section 3 we prove the existence of a skew symmetry of Blattner's formula that exists whenever $\Pi_{c} \neq \emptyset$. Thus, the condition that $\Pi_{c}=\emptyset$ is necessary for $B(\delta, \mu) \geq 0$ for all $\delta \in P_{+}(\mathfrak{k})$ and $\mu \in P(\mathfrak{k})$. In the situation where $\Pi_{c}=\emptyset$ we introduce the following:

Definition. We say that a semisimple Lie algebra is $\mathbf{b}$-positive if the Blattner formula corresponding to the $\mathbb{Z}_{2}$-gradation with $\Pi_{c}=\emptyset$ has the property that:

$$
B(\delta, \mu) \geq 0 \quad \text { for all } \delta \in P_{+}(\mathfrak{k}) \text { and } \mu \in P(\mathfrak{k}) .
$$

The terminology stems from the fact that a simple Lie algebra is $\mathbf{b}$-positive if and only if the coefficients of $\mathbf{b}(\delta)$ are nonnegative for all $\delta \in P_{+}(\mathfrak{k})$. Since the character of $L_{\mathfrak{k}}(\delta)$ can be written as a nonnegative integer combination of characters of $T$, we have that $\mathbf{b}(\delta)$ has nonnegative integer coefficients if $\mathbf{b}(0)$ does. Thus the question of $\mathbf{b}$-positivity reduces to the case of $\delta=0$. In Section [5, it is shown that the only b-positive simple Lie algebras are of type $A_{1}, A_{2}, A_{3}, B_{2}, C_{3}, D_{4}$ and $G_{2}$. We prove this result by examining the coefficients of $\mathbf{b}(0)$.

\section{Proof of the Main Result}

Proposition 2.1. For $\delta \in P_{+}(\mathfrak{k})$,

$$
\mathbf{b}(\delta)=\operatorname{ch} L_{\mathfrak{k}}(\delta) \frac{\prod_{\gamma \in \Phi_{c}^{+}}\left(1-e^{-\gamma}\right)}{\prod_{\gamma \in \Phi_{n c}^{+}}\left(1-e^{-\gamma}\right)},
$$


where $\operatorname{ch} L_{\mathfrak{k}}(\delta)$ denotes the character of $L_{\mathfrak{k}}(\delta)$.

Proof. From the definition of Blattner's formula we have:

$$
\mathbf{b}(\delta)=\sum_{\mu \in \mathfrak{h}^{*}} \sum_{w \in W_{\mathfrak{k}}}(-1)^{\ell(w)} Q(w(\delta+\rho)-\rho-\mu) e^{\mu} .
$$

First we make the substitution, $\mu=w(\delta+\rho)-\rho-\xi$, and reorganize the sum:

$$
\begin{aligned}
\mathbf{b}(\delta) & =\sum_{w \in W_{\mathfrak{k}}}(-1)^{\ell(w)} \sum_{\xi \in \mathfrak{h}^{*}} Q(\xi) e^{w(\delta+\rho)-\rho-\xi} \\
& =\sum_{w \in W_{\mathfrak{k}}}(-1)^{\ell(w)} e^{w(\delta+\rho)-\rho} \sum_{\xi \in \mathfrak{h}^{*}} Q(\xi) e^{-\xi} \\
& =\frac{\sum_{w \in W_{\mathfrak{k}}}(-1)^{\ell(w)} e^{w(\delta+\rho)-\rho}}{\prod_{\gamma \in \Phi_{n c}^{+}}\left(1-e^{-\gamma}\right)} \\
& =\frac{\sum_{w \in W_{\mathfrak{k}}}(-1)^{\ell(w)} e^{w(\delta+\rho)-\rho}}{\prod_{\gamma \in \Phi_{c}^{+}}\left(1-e^{-\gamma}\right)} \frac{\prod_{\gamma \in \Phi_{c}^{+}}\left(1-e^{-\gamma}\right)}{\prod_{\gamma \in \Phi_{n c}^{+}}\left(1-e^{-\gamma}\right)} .
\end{aligned}
$$

As is well known, the character may be expressed using Weyl's formula (see [4,8]) as in the following:

$$
\operatorname{ch} L_{\mathfrak{k}}(\delta)=\frac{\sum_{w \in W_{\mathfrak{k}}}(-1)^{\ell(w)} e^{w(\delta+\rho)-\rho}}{\prod_{\gamma \in \Phi_{c}^{+}}\left(1-e^{-\gamma}\right)} .
$$

The result allows us to compute the Blattner formula as follows:

$$
\sum_{\mu \in \mathfrak{h}^{*}} B(\delta, \mu) e^{\mu}=\operatorname{ch} L_{\mathfrak{k}}(\delta) \sum_{\nu \in \mathfrak{h}^{*}} B(0, \nu) e^{\nu} .
$$

Letting $\operatorname{ch} L_{\mathfrak{k}}(\delta)=\sum_{\gamma \in \mathfrak{h}^{*}} m_{\gamma} e^{\gamma}$ we obtain:

$$
\sum_{\mu \in \mathfrak{h}^{*}} B(\delta, \mu) e^{\mu}=\sum_{\gamma, \nu \in \mathfrak{h}^{*}} m_{\gamma} B(0, \nu) e^{\gamma+\nu} .
$$

Thus for $\delta \in P_{+}(\mathfrak{k})$ and $\mu \in P(\mathfrak{k})$ we have

$$
B(\delta, \mu)=\sum_{\gamma \in P(\mathfrak{k})} m_{\gamma} B(0, \mu-\gamma) .
$$

Note that the numbers $m_{\gamma}$ are weight multiplicities for the representation $L_{\mathfrak{k}}(\delta)$.

\section{3. (Skew-)Symmetries of Blattner's formula}

The main result of this section is:

Proposition 3.1. For $v \in W_{c}$ :

$$
\begin{array}{ll}
B(\delta, \mu)=B(\delta, v \cdot \mu) & \text { if } \ell(v) \text { is even, } \\
B(\delta, \mu)=-B(\delta, v . \mu) & \text { if } \ell(v) \text { is odd. }
\end{array}
$$

Although this is well known to experts, we include our proof as it requires very little technical machinery.

Definition. For $w \in W_{\mathfrak{g}}$ and $\xi \in \mathfrak{h}^{*}$, let $Q_{w}(\xi):=Q\left(w^{-1} \xi\right)$.

Lemma 3.1. If $w\left(\Phi_{n c}^{+}\right)=\Phi_{n c}^{+}$, then $Q_{w}=Q$. 
Proof. It is enough to show

$$
\sum_{\xi \in \mathfrak{h}^{*}} Q_{w}(\xi) e^{\xi}=\sum_{\xi \in \mathfrak{h}^{*}} Q(\xi) e^{\xi}
$$

which follows from the following calculation:

$$
\begin{aligned}
& \sum_{\xi} Q_{w}(\xi) e^{\xi}=\sum_{\xi} Q(\xi) e^{w(\xi)}=w\left(\sum_{\xi} Q(\xi) e^{\xi}\right) \\
& =w\left(\prod_{\alpha \in \Phi_{n c}^{+}}\left(1-e^{\alpha}\right)^{-1}\right)=\prod_{\alpha \in \Phi_{n c}^{+}}\left(1-e^{w(\alpha)}\right)^{-1} \\
& =\prod_{w^{-1}(\alpha) \in \Phi_{n c}^{+}}\left(1-e^{\alpha}\right)^{-1}=\prod_{\alpha \in w\left(\Phi_{n c}^{+}\right)}\left(1-e^{\alpha}\right)^{-1} \text {. }
\end{aligned}
$$

Lemma 3.2. For all $w \in W_{c}, w\left(\Phi_{n c}^{+}\right)=\Phi_{n c}^{+}$.

Proof. Note that $W_{c}$ is the Weyl group of a reductive Levi factor $\mathfrak{l}$ of a parabolic subalgebra $\mathfrak{q} \subseteq \mathfrak{g}$. We have a generalized triangular decomposition $\mathfrak{g}=\mathfrak{u}^{-} \oplus$ $\mathfrak{l} \oplus \mathfrak{u}^{+}$with $\mathfrak{q}=\mathfrak{l} \oplus \mathfrak{u}^{+}$. The noncompact root spaces contained in $\mathfrak{u}^{+}$are positive. Furthermore, all noncompact positive root spaces are contained in $\mathfrak{u}^{+}$because $\mathfrak{l} \subseteq \mathfrak{k}$. The Lie algebra $u^{+}$is an l-module, and therefore the weights are preserved by $W_{c}$. It is this fact that implies that $W_{c}$ takes positive noncompact roots to positive roots. We now need to show that $W_{c}$ takes noncompact roots to noncompact roots.

For roots $\beta \in \Phi_{n c}$ and $\alpha \in \Phi_{c}$, we have the formula $s_{\alpha}(\beta)=\beta-\frac{2(\alpha, \beta)}{(\alpha, \alpha)} \alpha$ with $\frac{2(\alpha, \beta)}{(\alpha, \alpha)}$ an integer. Thus, the reflection of a noncompact root across a hyperplane defined by a compact root is noncompact. The reflections generate $W_{c}$.

Proof of Proposition 3.1. Let $v \in W_{c}$ :

$$
B(\delta, \mu)=\sum_{w \in W_{\mathfrak{k}}}(-1)^{\ell(w)} Q(w \cdot \delta-\mu)=\sum_{w \in v\left(W_{\mathfrak{k}}\right)}(-1)^{\ell\left(v^{-1} w\right)} Q\left(\left(v^{-1} w\right) \cdot \delta-\mu\right) .
$$

By definition, $\ell(v)=\ell\left(v^{-1}\right)$. Combining this with the definition of the "dot" action we obtain:

$$
\begin{aligned}
B(\delta, \mu) & =(-1)^{\ell(v)} \sum_{w \in W_{\mathfrak{k}}}(-1)^{\ell(w)} Q\left(v^{-1} w(\delta+\rho)-\rho-\mu\right) \\
& =(-1)^{\ell(v)} \sum_{w \in W_{\mathfrak{k}}}(-1)^{\ell(w)} Q\left(v\left(v^{-1} w(\delta+\rho)-\rho-\mu\right)\right) .
\end{aligned}
$$

Next, we use the fact that $Q=Q_{v^{-1}}$ using Lemmas 3.1 and 3.2. The rest is a calculation:

$$
\begin{aligned}
B(\delta, \mu) & =(-1)^{\ell(v)} \sum_{w \in W_{\mathrm{k}}}(-1)^{\ell(w)} Q(w(\delta+\rho)-v(\mu+\rho)) \\
& =(-1)^{\ell(v)} \sum_{w \in W_{\mathrm{k}}}(-1)^{\ell(w)} Q(w(\delta+\rho)-\rho-v(\mu+\rho)+\rho) \\
& =(-1)^{\ell(v)} \sum_{w \in W_{\mathrm{k}}}(-1)^{\ell(w)} Q(w(\delta+\rho)-\rho-(v(\mu+\rho)-\rho)) \\
& =(-1)^{\ell(v)} \sum_{w \in W_{\mathrm{k}}}(-1)^{\ell(w)} Q(w \cdot \delta-v \cdot \mu)=(-1)^{\ell(v)} B(\delta, v \cdot \mu) .
\end{aligned}
$$




\section{The CASE OF $\mathrm{G}_{2}$}

The following is a complete calculation of $\mathbf{b}(0)$ for the case of the Lie algebra $\mathfrak{g}:=\mathrm{G}_{2}$ when $\Pi_{c}=\emptyset$. Let $\alpha$ and $\beta$ be a choice of noncompact simple roots for $\mathrm{G}_{2}$ with $\alpha$ long and $\beta$ short. The compact positive roots are $\Phi_{c}^{+}=\{\alpha+\beta, \alpha+3 \beta\}$, while the noncompact positive roots are $\Phi_{n c}^{+}=\{\alpha, \beta, \alpha+2 \beta, 2 \alpha+3 \beta\}$. Denote the $\Phi_{n c}^{+}$-partition function by $Q: \mathfrak{h}^{*} \rightarrow \mathbb{Z}$. We have:

$$
\mathbf{q}:=\sum_{\xi \in \mathfrak{h}^{*}} Q(\xi) e^{-\xi}=\prod_{\gamma \in \Phi_{n c}^{+}}\left(1-e^{-\gamma}\right)^{-1} .
$$

Let $x=e^{-\alpha}$ and $y=e^{-\beta}$. Thus,

$$
\mathbf{q}=\frac{1}{(1-x)(1-y)\left(1-x y^{2}\right)\left(1-x^{2} y^{3}\right)} .
$$

Let the simple reflection corresponding to $\alpha+\beta$ (resp. $\alpha+3 \beta)$ be $s_{1}$ (resp. $s_{2}$ ). We have four terms in Blattner's formula for $\delta=0$ :

$$
B(0, \mu)=Q(-\mu)-Q\left(s_{1} \rho-\rho-\mu\right)-Q\left(s_{2} \rho-\rho-\mu\right)+Q\left(s_{1} s_{2} \rho-\rho-\mu\right),
$$

and our goal will be to close the sum $\mathbf{b}(0):=\sum_{\mu \in \mathfrak{h}^{*}} B(0, \mu) e^{\mu}$. We will do this by multiplying by $e^{\mu}$ and summing over $\mu$ for each of the four terms. Observe that:

$$
\sum_{\xi \in \mathfrak{h}^{*}} Q(\xi) e^{-\xi}=\sum_{\xi \in \mathfrak{h}^{*}} Q(-\xi) e^{\xi}
$$

Thus, $\mathbf{q}=\sum_{\xi \in \mathfrak{h}^{*}} Q(-\xi) e^{\xi}$. Next consider the sum:

$$
T_{1}:=\sum_{\xi \in \mathfrak{h}^{*}} Q\left(s_{1} \rho-\rho-\xi\right) e^{\xi} .
$$

We make the substitution $-\mu=s_{1} \rho-\rho-\xi$ so that the above sum becomes:

$$
\sum_{\mu \in \mathfrak{h}^{*}} Q(-\mu) e^{s_{1} \rho-\rho+\mu}=e^{s_{1} \rho-\rho} \sum_{\mu \in \mathfrak{h}^{*}} Q(-\mu) e^{\mu} .
$$

Thus the above sum is equal to $e^{s_{1} \rho-\rho} \mathbf{q}$, which we denote by $\mathbf{T}_{\mathbf{1}}$. Similarly, we set $\mathbf{T}_{\mathbf{2}}:=e^{s_{2} \rho-\rho} \mathbf{q}$ and $\mathbf{T}_{\mathbf{3}}:=e^{s_{1} s_{2} \rho-\rho} \mathbf{q}$, and $\mathbf{T}_{\mathbf{0}}:=\mathbf{q}$. Thus, $\mathbf{b}(0)=\mathbf{T}_{\mathbf{0}}-\mathbf{T}_{\mathbf{1}}-\mathbf{T}_{\mathbf{2}}+\mathbf{T}_{\mathbf{3}}$.

Now we write the above in terms of $x$ and $y$. Note that $\rho=\alpha+2 \beta, s_{1} \rho=\beta$, $s_{2} \rho=-\beta$, and from these we can easily see:

$$
e^{s_{1} \rho-\rho}=x y, \quad e^{s_{2} \rho-\rho}=x y^{3}, \quad \text { and } \quad e^{s_{1} s_{2} \rho-\rho}=x^{2} y^{4} .
$$

Putting everything together we see $\mathbf{b}(0)=(1-x y)\left(1-x y^{3}\right) \mathbf{q}$. Or equivalently, if $\mu=-k \alpha-\ell \beta$, then the value of $B(0, \mu)$ is the coefficient of $x^{k} y^{\ell}$ in

$\mathbf{b}(0)=\frac{(1-x y)\left(1-x y^{3}\right)}{(1-x)(1-y)\left(1-x y^{2}\right)\left(1-x^{2} y^{3}\right)}=\frac{1}{(1-x)\left(1-x y^{2}\right)}+\frac{y}{(1-y)\left(1-x^{2} y^{3}\right)}$.

Note that in the latter expression, it is clear that the coefficients in the series are positive. The positivity of the coefficients of $\mathbf{b}(\delta)$ follows from the positivity of the coefficients of $\mathbf{b}(0)$. The question of positivity for a general semisimple Lie algebra will be addressed in Section 5 .

It is important to note that as we change $\Pi_{c}$, the value of $\mathbf{b}(0)$ changes as well. For example, when $\Pi_{c}=\{\beta\}$,

$$
\mathbf{b}(0)=\frac{(1-y)\left(1-x^{2} y^{3}\right)}{(1-x)(1-x y)\left(1-x y^{2}\right)\left(1-x y^{3}\right)}=\frac{1}{(1-x)\left(1-x y^{2}\right)}-\frac{y}{(1-x y)\left(1-y^{3} x\right)},
$$



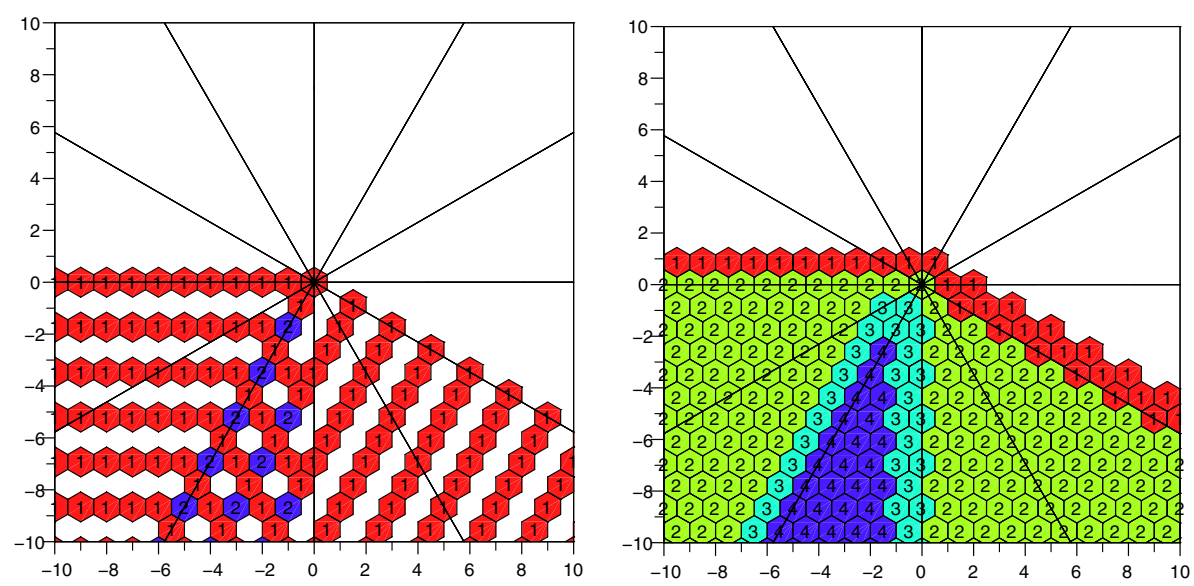

Figure $1 . \mathbf{b}(\delta)$ when $\Pi_{c}=\emptyset$ with $\delta=0$ (left) and $\delta=\rho$ (right)
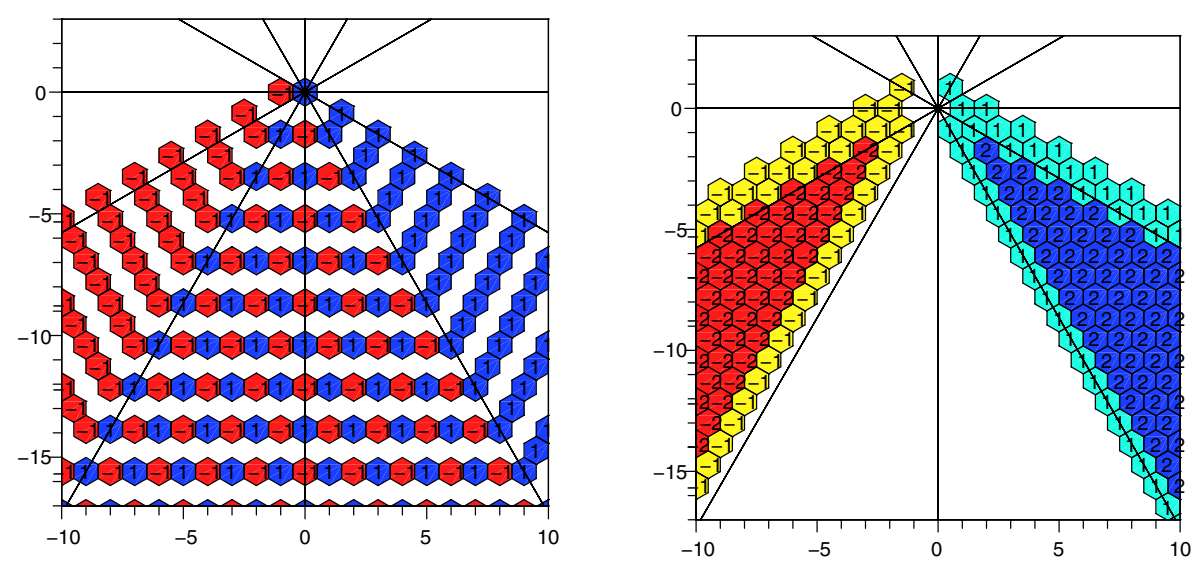

Figure 2. $\mathbf{b}(\delta)$ when $\Pi_{c}=\{\beta\}$ with $\delta=0$ (left) and $\delta=\rho$ (right)

which we can easily see does not have nonnegative integer coefficients. We leave it as an exercise to the reader to compute $\mathbf{b}(0)$ when $\Pi_{c}=\{\alpha\}$.

The generating functions for other $\delta$ involve multiplying the above product by a polynomial in $x^{ \pm \frac{1}{2}}, y^{ \pm \frac{1}{2}}$ that represents the character of the corresponding irreducible finite-dimensional representation of $\mathfrak{k}=\mathfrak{s o}_{4}$.

One can plot the coefficients of this formal series, as we do next. In all pictures we have labeled the scale on the axes and normalized the short root $(\beta)$ to have length 1 and be positioned at 3 o'clock. The long root $\alpha$ is at 10 o'clock.

The first two images (Figure 1) are for the case with $\Pi_{c}=\emptyset$ (generic) and correspond to $\delta=0$ and $\delta$ the highest weight of the standard 4-dimensional representation of $\mathfrak{s o}_{4}$. We also display the same two $\delta$ 's in the case when $\Pi_{c}=\{\beta\}$ (Borel-de Siebenthal) in Figure 2. These latter two figures clearly display a skewsymmetry addressed in Section 3 . 


\section{5. b-POSITIVE SIMPLE LIE ALGEBRAS}

Proposition 5.1. The only $\mathbf{b}$-positive simple Lie algebras are of type $\mathrm{A}_{1}, \mathrm{~A}_{2}, \mathrm{~A}_{3}$, $\mathrm{B}_{2}, \mathrm{C}_{3}, \mathrm{D}_{4}$ and $\mathrm{G}_{2}$.

To prove the proposition we use the following lemma to exclude the other cases.

Lemma 5.1. Let $\mathfrak{l}$ be the semisimple Levi factor of a parabolic subalgebra of $\mathfrak{g}$. If $\mathfrak{g}$ is $\mathbf{b}$-positive, then $\mathfrak{l}$ is also $\mathbf{b}$-positive.

Proof. It is a consequence of the main proposition that for any rank $r$ simple Lie algebra $\mathfrak{g}$ we may express $\mathbf{b}(0)$ as a rational function in $x_{1}, \ldots, x_{r}$ where $x_{i}=e^{-\alpha_{i}}$ (recall that $\alpha_{i}$ is the $i^{\text {th }}$-simple root for $\mathfrak{g}$ ). For any $j$, if we set $x_{j}=0$ in $\mathbf{b}(0)$, then the resulting expression is $\mathbf{b}(0)$ for a semisimple subalgebra of $\mathfrak{g}$. It is not hard to see that this subalgebra is the Levi factor of the maximal parabolic subalgebra of $\mathfrak{g}$ corresponding to $\alpha_{j}$. More generally, let $S \subseteq \Pi$. If we set $x_{i}=0$ in $\mathbf{b}(0)$ for all $\alpha_{i} \in S$, then the resulting expression is $\mathbf{b}(0)$ for the Levi subalgebra, $\mathfrak{l}_{S}$, of the corresponding parabolic subalgebra. Note that the terms in the series expansion for $\mathbf{b}(0)$ for $\mathfrak{l}_{S}$ are also terms in the series expansion of $\mathbf{b}(0)$ for $\mathfrak{g}$. Thus a negative coefficient in the former implies a negative coefficient in the latter.

The proof of Proposition 5.1 involves a case-by-case analysis using the main proposition expressing $\mathbf{b}(0)$ as a product, Lemma 5.1 and the computer algebra package, MAPLE. Recall that the b-positivity of $\mathrm{G}_{2}$ was proved in Section 4 .

5.1. Type A. For $\mathfrak{s l}_{2}$, we have $\mathbf{b}(0)=\frac{1}{1-x_{1}}$. For $\mathfrak{s l}_{3}$ :

$$
\mathbf{b}(0)=\frac{1-x_{1} x_{2}}{\left(1-x_{1}\right)\left(1-x_{2}\right)}=1+\frac{x_{1}}{1-x_{1}}+\frac{x_{2}}{1-x_{2}} .
$$

For $\mathfrak{s l}_{4}$ :

$$
\begin{aligned}
\mathbf{b}(0) & =\frac{\left(1-x_{1} x_{2}\right)\left(1-x_{2} x_{3}\right)}{\left(1-x_{1}\right)\left(1-x_{2}\right)\left(1-x_{3}\right)\left(1-x_{1} x_{2} x_{3}\right)} \\
& =\frac{1}{\left(1-x_{3}\right)\left(1-x_{1}\right)}+\frac{x_{2}}{\left(1-x_{2}\right)\left(1-x_{1} x_{2} x_{3}\right)} .
\end{aligned}
$$

The partial fraction on the right of each of these examples establishes that the coefficients are indeed positive. Next we consider $\mathfrak{s l}_{5}$ where we have a negative result. First we note:

$$
\mathbf{b}(0)=\frac{\left(1-x_{1} x_{2}\right)\left(1-x_{2} x_{3}\right)\left(1-x_{3} x_{4}\right)\left(1-x_{1} x_{2} x_{3} x_{4}\right)}{\left(1-x_{1}\right)\left(1-x_{2}\right)\left(1-x_{3}\right)\left(1-x_{4}\right)\left(1-x_{1} x_{2} x_{3}\right)\left(1-x_{2} x_{3} x_{4}\right)},
$$

for $\mathfrak{s l}_{5}$. We expand in a formal power series and observe that the coefficient of $x_{1} x_{2}^{2} x_{3}^{2} x_{4}$ is -1 . This means that $\mathbf{b}$-positivity fails for this example. We then also see failure of b-positivity for any simple Lie algebra which has $\mathrm{A}_{4}$ as a Levi factor of a parabolic subalgebra. Thus, we exclude all higher-rank type A examples as well as $\mathrm{B}_{n}(n \geq 5), \mathrm{C}_{n}(n \geq 5), \mathrm{D}_{n}(n \geq 5), \mathrm{E}_{6}, \mathrm{E}_{7}$ and $\mathrm{E}_{8}$.

5.2. Type B. We only need to examine $\mathfrak{s o}_{5}$ and $\mathfrak{s o}_{7}$. For $\mathfrak{s o}_{5}$ we have

$$
\begin{aligned}
\mathbf{b}(0) & =\frac{1-x_{1} x_{2}}{\left(1-x_{1}\right)\left(1-x_{2}\right)\left(1-x_{1} x_{2}^{2}\right)} \\
& =\frac{1}{\left(1-x_{2}^{2}\right)\left(1-x_{1}\right)}+\frac{x_{2}}{\left(1-x_{2}^{2}\right)\left(1-x_{1} x_{2}^{2}\right)} .
\end{aligned}
$$


Thus the coefficients of the series expansion are nonnegative.

Now consider $\mathfrak{s o}_{7}$ :

$$
\mathbf{b}(0)=\frac{\left(1-x_{1} x_{2}\right)\left(1-x_{2} x_{3}\right)\left(1-x_{1} x_{2} x_{3}^{2}\right)}{\left(1-x_{1}\right)\left(1-x_{2}\right)\left(1-x_{3}\right)\left(1-x_{1} x_{2} x_{3}\right)\left(1-x_{2} x_{3}^{2}\right)\left(1-x_{1} x_{2}^{2} x_{3}^{2}\right)} .
$$

Upon expansion we see that the coefficient of $x_{1}^{2} x_{2}^{3} x_{3}^{3}$ is -1 . Thus, we may exclude this and higher-rank type $\mathrm{B}$ examples as they have $B_{3}$ as the Levi factor of a parabolic subalgebra. In particular, we may exclude $B_{4}$, which is the only type $\mathrm{B}$ example that has not yet been excluded. We also may exclude $\mathrm{F}_{4}$ for the same reason.

5.3. Type C. We must examine $\mathbf{b}(0)$ for $\mathfrak{s p}_{6}$ and $\mathfrak{s p}_{8}$ as these are the only examples not yet addressed. For $\mathfrak{s p}_{6}$ (i.e. $C_{3}$ ), we have

$$
\mathbf{b}(0)=\frac{\left(1-x_{1} x_{2}\right)\left(1-x_{2} x_{3}\right)\left(1-x_{1} x_{2}{ }^{2} x_{3}\right)}{\left(1-x_{1}\right)\left(1-x_{2}\right)\left(1-x_{3}\right)\left(1-x_{1} x_{2} x_{3}\right)\left(1-x_{2}{ }^{2} x_{3}\right)\left(1-x_{1}{ }^{2} x_{2}{ }^{2} x_{3}\right)} .
$$

The coefficients are positive as the above expression is equal to

$$
\begin{gathered}
\frac{1}{\left(1-x_{1}\right)\left(1-x_{2}^{2} x_{3}\right)}+\frac{x_{3}}{\left(1-x_{2}^{2} x_{3}\right)\left(1-x_{1}^{2} x_{2}^{2} x_{3}\right)\left(1-x_{3}\right)}+\frac{x_{2}}{\left(1-x_{2}^{2} x_{3}\right)\left(1-x_{1}^{2} x_{2}^{2} x_{3}\right)\left(1-x_{2}\right)} \\
+\frac{x_{1} x_{3}}{\left(1-x_{1}\right)\left(1-x_{3}\right)\left(1-x_{1}^{2} x_{2}^{2} x_{3}\right)}+\frac{x_{1}^{2} x_{2}^{3} x_{3}^{2}}{\left(1-x_{2}^{2} x_{3}\right)\left(1-x_{1}^{2} x_{2}^{2} x_{3}\right)\left(1-x_{1} x_{2} x_{3}\right)} .
\end{gathered}
$$

However, we do not have $\mathbf{b}$-positivity for $\mathfrak{s p}_{8}$, as we see -1 as the coefficient of $x_{1} x_{2}^{3} x_{3}^{3} x_{4}^{2}$ in $\mathbf{b}(0)=\frac{N U M}{D E N}$ where:

$$
\begin{aligned}
N U M:= & \left(1-x_{1} x_{2}\right)\left(1-x_{2} x_{3}\right)\left(1-x_{3} x_{4}\right)\left(1-x_{1} x_{2} x_{3} x_{4}\right) \\
& \times\left(1-x_{2} x_{3}{ }^{2} x_{4}\right)\left(1-x_{1} x_{2}{ }^{2} x_{3}{ }^{2} x_{4}\right), \\
D E N:= & \left(1-x_{1}\right)\left(1-x_{2}\right)\left(1-x_{3}\right)\left(1-x_{4}\right)\left(1-x_{1} x_{2} x_{3}\right)\left(1-x_{2} x_{3} x_{4}\right) \\
& \times\left(1-x_{3}{ }^{2} x_{4}\right)\left(1-x_{1} x_{2} x_{3}{ }^{2} x_{4}\right)\left(1-x_{2}{ }^{2} x_{3}{ }^{2} x_{4}\right)\left(1-x_{1}{ }^{2} x_{2}{ }^{2} x_{3}{ }^{2} x_{4}\right) .
\end{aligned}
$$

5.4. Type D. The only case left is $\mathfrak{s o}_{8}$, where we have $\mathbf{b}(0)=\frac{N U M}{D E N}$ where

$$
\begin{aligned}
\mathrm{NUM}= & \left(1-x_{1} x_{2}\right)\left(1-x_{2} x_{3}\right)\left(1-x_{2} x_{4}\right)\left(1-x_{1} x_{2} x_{3} x_{4}\right), \\
\mathrm{DEN}= & \left(1-x_{1}\right)\left(1-x_{2}\right)\left(1-x_{3}\right)\left(1-x_{4}\right)\left(1-x_{1} x_{2} x_{3}\right)\left(1-x_{1} x_{2} x_{4}\right) \\
& \times\left(1-x_{2} x_{3} x_{4}\right)\left(1-x_{1} x_{2}{ }^{2} x_{3} x_{4}\right) .
\end{aligned}
$$

We see that

$$
\begin{aligned}
\mathbf{b}(0)= & \frac{1}{\left(1-x_{1} x_{2}^{2} x_{3} x_{4}\right)\left(1-x_{1}\right)\left(1-x_{3}\right)\left(1-x_{4}\right)} \\
& +\frac{x_{2}}{\left(1-x_{1} x_{2} x_{4}\right)\left(1-x_{2}\right)\left(1-x_{2} x_{3} x_{4}\right)\left(1-x_{1} x_{2} x_{3}\right)} .
\end{aligned}
$$

Thus the coefficients of the series expansion are nonnegative integers.

\section{REFERENCES}

[1] Thomas J. Enright, On the fundamental series of a real semisimple Lie algebra: Their irreducibility, resolutions and multiplicity formulae, Ann. of Math. (2) 110 (1979), no. 1, 1-82. MR.541329 (81a:17003)

[2] Thomas J. Enright and V. S. Varadarajan, On an infinitesimal characterization of the discrete series, Ann. of Math. (2) 102 (1975), no. 1, 1-15. MR0476921 (57:16472)

[3] Thomas J. Enright and Nolan R. Wallach, The fundamental series of representations of a real semisimple Lie algebra, Acta Math. 140 (1978), no. 1-2, 1-32. MR0476814 (57:16368) 
[4] R. Goodman and N.R. Wallach, Representations and invariants of the classical groups, Cambridge University Press, Cambridge, 1998. MR.1606831 (99b:20073)

[5] B. Gross and N. Wallach, Restriction of small discrete series representations to symmetric subgroups, The mathematical legacy of Harish-Chandra (Baltimore, MD, 1998), Proc. Sympos. Pure Math., vol. 68, Amer. Math. Soc., Providence, RI, 2000, pp. 255-272. MR 1767899 (2001f:22042)

[6] Harish-Chandra, Discrete series for semisimple Lie groups. II. Explicit determination of the characters, Acta Math. 116 (1966), 1-111. MR0219666 (36:2745)

[7] Henryk Hecht and Wilfried Schmid, A proof of Blattner's conjecture, Invent. Math. 31 (1975), no. 2, 129-154. MR0396855(53:715)

[8] Anthony W. Knapp, Lie groups beyond an introduction, Second edition, Progress in Mathematics, vol. 140, Birkhäuser Boston Inc., Boston, MA, 2002. MR1920389 (2003c:22001)

[9] Ivan Penkov and Gregg Zuckerman, Generalized Harish-Chandra modules with generic minimal k-type, Asian J. Math. 8 (2004), no. 4, 795-811. MR2127949(2005k:17007)

[10] Wilfried Schmid, $L^{2}$-cohomology and the discrete series, Ann. of Math. (2) 103 (1976), no. 2, 375-394. MR0396856 (53:716)

[11] David A. Vogan Jr., Representations of real reductive Lie groups, Progress in Mathematics, vol. 15, Birkhäuser, Boston, MA, 1981. MR632407|(83c:22022)

[12] Nolan R. Wallach, Real reductive groups. I, Pure and Applied Mathematics, vol. 132, Academic Press Inc., Boston, MA, 1988. MR929683 (89i:22029)

[13] _ Real reductive groups. II, Pure and Applied Mathematics, vol. 132, Academic Press Inc., Boston, MA, 1992. MR:1170566 (93m:22018)

[14] Gregg J. Zuckerman, Coherent translation of characters of semisimple Lie groups, Proceedings of the International Congress of Mathematicians (Helsinki, 1978), Acad. Sci. Fennica, Helsinki, 1980, pp. 721-724. MR.562678 (81f:22025)

Department of Mathematical Sciences, University of Wisconsin-Milwaukee, P.O. Box 0413, Milwaukee, Wisconsin 53201-0413

E-mail address: jw@uwm.edu

Department of Mathematics, Yale University, P.O. Box 208283; New Haven, ConNECTICUT 06520-8283

E-mail address: gregg.zuckerman@yale.edu 